\title{
Numerical modeling of the nucleation of facets ahead of a primary crack under mode I+III loading
}

\author{
Aurélien Doitrand · Dominique Leguillon
}

Received: date / Accepted: date

\begin{abstract}
A numerical study of crack front segmentation under mode I+III loading is proposed. Facets initiation ahead of a parent crack is predicted through a tridimensional application of the coupled criterion. Crack initiation shape, orientation and spacing are determined for any mode mixity ratio by coupling a stress and an energy criterion using matched asymptotic expansions. The stress and the energy conditions are computed through a 3D finite element modeling of a periodic network of facets ahead of the parent crack. Facets initiation shape, loading and spacing depend on the blunted parent crack tip radius. A good estimate of facet orientations is obtained based on the direction of maximum tensile stress. The facet shapes, determined using the stress isocontours, are qualitatively similar to those observed experimentally. The order of magnitude of numerical predictions of facets spacing is very close to experimental measurements.
\end{abstract}

Keywords Mode I+III loading - Crack front fragmentation · Finite Fracture Mechanics · Coupled criterion

\section{Introduction}

Crack growth under combined mode I+III loading has been widely studied over the past decades. Under such

Aurélien Doitrand

Safran Aircraft Engines, Rond-point René Ravaud, 77550

Moissy-Cramayel, France

Tel.: +33 160596518

E-mail: aurelien.doitrand@gmail.com

Dominique Leguillon

Institut Jean le Rond d'Alembert, Sorbonne Université, Centre National de la Recherche Scientifique, UMR 7190, F-75005 Paris, France loading combination, the crack tends to rotate around the direction of propagation in order to reduce mode III and reach a pure mode I situation. This is achieved by a fragmentation of the initial crack into multiple daughter cracks (also called facets). Once initiated, some of these facets grow and coalesce so as to form a stepped fractured surface that becomes coarser as the crack grows [26]. Mode I+III crack patterns have been investigated experimentally for some years. Since the pioneering works of Sommer [29] and Knauss [28] who studied facet nucleation respectively in glass and in polymer, mode I+III crack patterns have been observed in various studies such as fault formation [5], cracks in rocks [33], in PMMA [1,12] or in plexiglass [3].

The geometrical description of facets is generally performed by observing post-mortem specimens in which the initial crack has segmented into daughter cracks that have already propagated and possibly coalesced. Cambonie and Lazarus [2] proposed a systematic method to quantify the geometry of the facets from post-mortem specimens by profilometry. They determined the distance between the facets and their twisting angles as a function of the distance to the parent crack front. Eberlein et al. [11] performed Arcan tests with CTSRspecimen under mode I+III fatigue loading so as to study the crack front segmentation profile using postmortem specimens. They determined the influence of the amount of mode III on some geometrical features of the segmented crack front such as facet angle, projected facet length, facet distance and bridging width. In order to quantify more in depth facet nucleation, Pham and Ravi-Chandar [24] designed a test so as to load a specimen in mode I+III through the combination of a wedge load and of a confining load resulting in a global compressive stress field ahead of the crack front. Multiple facet nucleation was observed by stop- 
ping the test just after crack initiation, which particularly highligthed that the facet coalescence does not happen during initiation, but at a later stage of crack growth. Mittelman and Yosibash $[22,32]$ carried out experiments on inclined V-notched specimens of PMMA, Graphite and ceramics under mode I+III loading, and concluded that facet initiation orientation can be determined from stress considerations.

Leblond et al. $[13,14]$ proposed a theoretical analysis of the instability of the crack front and the growth of the facets under mode I+III loading, which was also studied numerically by several authors, mainly from a macroscopic point of view. Using the modified virtual crack closure integral method, Dhondt et al. [6], Buchholz et al. [1] and Lazarus et al. [12] proposed finite element (FE) simulations of crack propagation in different configurations such as three point bending of specimens with a tilted initial crack . They qualitatively well reproduced the progressive crack rotation with respect to its initial direction until it reaches a pure mode I situation. Citarella and Buchholz [4] showed the consistency of the dual boundary element modeling for modeling crack propagation under mode I+III loading compared to FE modeling. However, these approachs involve a progressive twist of the crack, and are unable to take into account the initial crack front segmentation into facets. Pons and Karma [26] and Chen et al. [3] simulated crack growth based on a continuum phase field method, which allows reproducing the facet coarsening under mode I+III loading.

Facet nucleation was studied numerically by Pham and Ravi-Chandar [23] using the boundary element simulation. The stress intensity factors $K_{\mathrm{I}}$ and $K_{\mathrm{III}}$ along the parent crack front for which a first daughter crack has already initiated were computed, allowing the determination of a range for the spacing between facets corresponding to the locations maximizing $K_{\mathrm{I}}$ and minimizing $K_{\text {III }}$. Pham and Ravi-Chandar concluded that the distance between facets was dictated either by the fracture process zone for a natural crack, or by the local radius of curvature for a machined crack. The same authors [24] also highlited the shielding of some daughter cracks resulting from the propagation of neighboring cracks, hence leading to facet coarsening as the crack grows. These models allow the study of daughter crack initiation, after the nucleation of the first facet which has not been investigated until now.

The objective of the work is the prediction of daughter crack initiation ahead of a primary crack under mode I+III loading. In section 2, some experimental data and observations about facet nucleation are presented in order to justify the modeling hypotheses. The crack initiation prediction method, namely the coupled criterion, is presented in Section 3. In Section 4, daughter crack initiation is modeled by FE, and numerical prediction of facet nucleation are presented and confronted to some experimental data in Section 5. Throughout this paper, crack and facet are synonymous. When the main crack is under consideration, the denomination parent crack is used.

\section{Experimental observations and modeling hypotheses}

Crack rotation aiming at reaching a pure mode I situation during crack propagation under mode I+III loading usually occurs due to the parent crack front segmentation into multiples facets [1-3,23,24]. However, Eberlein et al. [11] showed experimentally that in some cases, below a certain $K_{\text {III }} / K_{\text {I }}$ ratio threshold, no facet formation are observed and the crack grows continuously. This phenomenon was not observed by Pham and Ravi-Chandar [23,24], who highlighted facet formation for ratios as small as $K_{\mathrm{III}} / K_{\mathrm{I}}=0.001$. Herein, only crack front segmentation into facets is adressed, whereas the following continuous crack growth is beyond the scope of this work.

Experimental observations under mode I+III loading show simultaneous initiation of several facets ahead of the parent crack. These cracks initiate forming a "nice nearly periodic pattern" [23], all cracks being (i) oriented with similar angles with respect to the parent crack $[3,23,24]$, (ii) separated by almost the same distance $[2,11,24]$ and (iii) having almost the same size $[3,24]$. Moreover, the nucleated facets are nearly planar $[2,24]$, except the crack tip that is gently curved [3]. In this plane, the initiation crack exhibits a nearly elliptic crack shape [24]. Several approaches exist in order to determine the crack orientation such as, e.g., analytical formulae $[12,25]$. In this work, we will assume that the normal to the crack plane is determined by the direction of the maximum tensile stress at the parent crack tip. Experimentally, mode I+III loading also includes a portion of mode II, especially near the free edges [11], which will be ignored in the proposed modeling.

Given the experimental observations described previously, the modeling hypotheses assumed in this work are the following :

o The imposed loading is a combination of mode I and mode III, without any mode II. It is independent of the curvilinear abscissa along the crack front.

o Aa a consequence, an infinite network of periodic cracks is modeled, which is representative of facet initiation far from free edges. 
o Facets extend in a plane during the nucleation phase, hence the gently curved crack tip is not taken into account.

o The crack angle with respect to the parent crack is determined as the one maximizing the tensile stress ahead of the parent crack tip.

o In the crack plane, no a priori hypothesis is made on the crack shape, which is directly determined based on the normal stress field, as explained in Section 3.4, following the approach proposed by Doitrand and Leguillon [9].

\section{The coupled criterion}

\subsection{Stress and energy conditions}

According to the coupled criterion (CC) [15], crack initiation requires two separate conditions that must be fulfilled. On the one hand, the stress state prior to crack initiation must be high enough, and on the other hand, sufficient energy must be released by the crack opening. Both conditions can be gathered to predict crack initiation.

The energy condition of the coupled criterion is obtained through an energy balance between an elastic state prior to crack initiation and after the nucleation of a crack of surface $S$ (Eq. 1).

$\delta W_{\mathrm{p}}+\delta W_{\mathrm{k}}+G_{\mathrm{c}} S=0$

where $\delta W_{\mathrm{p}}$ is the change in potential energy, $\delta W_{\mathrm{k}}$ the change in kinetic energy and $G_{\mathrm{c}}$ the material toughness. A quasi-static initial state leads to a production of kinetic energy $\left(\delta W_{\mathrm{k}} \geq 0\right)$, which results in the energy condition for crack initiation (Eq. 2).

$G^{\text {inc }}=-\frac{\delta W_{\mathrm{p}}}{S} \geq G_{\mathrm{c}}$

The stress condition of the CC compares the tensile stress acting in the direction orthogonal to the crack to the tensile strength $\sigma_{\mathrm{c}}(\mathrm{Eq} 3)$.

$\sigma \geq \sigma_{c}$ on the presupposed whole crack surface $S$

Combining both conditions (2) and (3) allows the determination of the initiation crack surface and loading level. As mentionned in Section 2, the shape of the facets is determined by the isocontours of the tensile stress, ensuring the stress condition to be fulfilled on the whole surface. When $S$ is small the isocontours are roughly homothetic curves, each one being defined by its extension along a choosen direction.

\subsection{Crack initiation ahead of a parent crack}

Experimental investigations of crack initiation and growth under mode I+III usually involve specimens with a blunted parent crack tip [24]. Crack initiation ahead of this parent crack tip depends on the crack tip radius, which can be considered as a characteristic length of the problem. The coupled criterion has been successfully applied to crack initiation prediction at blunted notches under mode I loading $[21,19,27]$, using matched asymptotics expansions in a $2 \mathrm{D}$ framework. This method consists in determining the displacement field for two problems, one far from the crack (the outer expansion) and one near it (the inner expansion), and then determine matching conditions in an intermediate zone (close to the crack in the outer problem and far from it for the inner problem) where both solutions are valid. The application of matched asymptotic expansions to crack initiation prediction at blunted notches under pure mode I loading using the coupled criterion is detailed in [19]. In the following, the choice of the inner domain and the method employed to solve the coupled criterion in pure mode I or in mixed mode I+III loading is described.

\subsection{Pure mode I loading}

Regardless of the blunting, in the outer domain spanned by the space variables $x_{i}(i=1,3)$, the crack is sharp (see the Appendix). The inner domain is obtained by dilatation of the space variables compared to a characteristic length of the problem. Classically [15], in the case of a sharp parent crack, the dilatation is carried out with respect to the crack extension $l$, whereas in the case of a blunted parent crack tip it can be performed either with respect to the crack extension $l$ or the crack tip radius $R$, provided that both lengths are of the same order of magnitude, property which is checked afterwards. For the sake of ease of FE mesh generation, we choose to perfom the dilatation with respect to the crack tip radius to obtain the inner domain. Therefore, the inner domain is an unbounded media spanned by the space variables $y_{i}=x_{i} / R$, containing a semi-infinite crack which root radius is equal to 1 , and a crack extension of dimensionless length $\lambda=l / R$. Leguillon et $a l$. [19] determined the change in potential energy from calculations in the inner domain (Eq. 4) in a problem under plane strain assumption, $e$ being the thickness of the specimen:

$$
\left\{\begin{array}{l}
-\delta W_{\mathrm{p}}=K_{\mathrm{I}}^{2} R(B(\lambda)-B(0)) e \\
G^{\text {inc }}=-\frac{\delta W_{\mathrm{p}}}{S}=-\frac{\delta W_{\mathrm{p}}}{l e}=K_{\mathrm{I}}^{2} \frac{B(\lambda)-B(0)}{\lambda}
\end{array}\right.
$$


where $K_{\mathrm{I}}$ is the stress intensity factor at the crack tip in the outer domain (keep in mind that in this domain, the crack is sharp) and $B(\lambda)$ can be computed by FE modeling calculations on the inner domain with various values of $\lambda$ (cf. Section 4.1). Of course, the FE calculations of $B(\lambda)$ cannot be performed on an infinite domain. Therefore, the inner domain is artificially bounded at a large distance from the crack. In the case of pure mode I loading, the crack initiates and grows in the plane of the parent crack. Therefore, the domain corresponding to the inner problem for FE calculations is defined as a cylinder of any thickness and outer radius $\Pi$ embedding a parent crack with a blunted tip of radius 1 ( $c f$. Fig. 1a).

Using Matched asymptotic expansions in the 2D case, Leguillon et al. [19] determined the problem to solve in order to obtain the loading level $K_{\text {Ic }}^{\text {app }}$ and the length $l_{\mathrm{c}}$ of a crack initiating ahead of a blunted crack tip of radius $R$ (Eq. 5 and 6$)$.

$\frac{1}{\tilde{\sigma}\left(\lambda_{c}\right)^{2}} \frac{B\left(\lambda_{c}\right)-B(0)}{\lambda_{c}}=\frac{1}{R} \frac{G_{\mathrm{c}}}{\sigma_{\mathrm{c}}^{2}}$

where $\lambda_{c}=l_{c} / R$ is the non-dimensional initiation crack length, $\tilde{\sigma}(\lambda)$ stands for the non-dimensional tensile stress in the inner domain at a distance $\lambda$ from the blunted tip prior to crack initiation, and $\frac{B(\lambda)-B(0)}{\lambda}$ is computed by $\mathrm{FE}$ for several values of $\lambda$ (refer to [19] and to the Appendix for more precise definitions of these terms).

$K_{\mathrm{Ic}}^{\mathrm{app}}=\sqrt{\frac{\lambda_{c} G_{\mathrm{c}}}{B\left(\lambda_{c}\right)-B(0)}}$

The same approach can be applied to the 3D case treated herein, assuming that the initiation crack front is parallel to the parent crack front, the $3 \mathrm{D}$ problem being reduced to a $2 \mathrm{D}$ problem in the plane orthogonal to $y_{3}$.

\subsection{Mixed mode I+III}

Under mode I+III loading, facets that initiate ahead of the parent crack form an angle $\phi$ (see Figure 1) with this parent crack. In the following, the mode mixity ratio is denoted $\beta$ (Eq. 7 ), where $K_{\mathrm{I}}$ and $K_{\text {III }}$ are the mode I and III stress intensity factors in the outer domain (see the Appendix).

$\beta=\frac{K_{\mathrm{III}}}{K_{\mathrm{I}}+K_{\mathrm{III}}}$

Following the hypotheses described in Section 2, the choosen inner domain is a cylinder of outer radius $\Pi$ and thickness $\epsilon$ containing a semi-infinite parent crack with a blunted tip of radius unity in which a single crack initiates. Periodic boundary conditions with period $\epsilon=e / R$ are prescribed along $y_{3}$-direction in order to model the simultaneous initiation of several cracks of similar size and orientation, regularly spaced ahead of the parent crack, $\epsilon$ being the dimensionless spacing (unknown up to now) between facets. The possible initiation cracks, whose modeling is described in Section 4.1, exhibit a complex 3D shape, which may no longer be described by a length, but rather by some parameters (and possibly an infinite number, as evoked in $[7,30])$. In order to overcome this difficulty, Doitrand and Leguillon [9] proposed to determine the possible crack shapes based on the stress isocontours. The main advantage of this method is that the possible crack shapes can be described by a single parameter, e.g., their surface $S$ or their extension $l$ along $\left(O y_{1}\right)$ axis, assuming that the crack surface can be written $S=\alpha l^{2}$ (where $\alpha$ is a scaling coefficient). Once the dilatation of space variables (detailed in the Appendix) has been performed, equation (4) rewrites (powers differ slightly from $2 \mathrm{D}$ to $3 \mathrm{D}$, refer to [17] and to the Appendix):

$\left\{\begin{array}{l}-\delta W_{\mathrm{P}}=K_{\mathrm{I}}^{2} R^{2}(B(\lambda)-B(0)) \\ G^{\mathrm{inc}}=K_{\mathrm{I}}^{2} \frac{B(\lambda)-B(0)}{\alpha \lambda^{2}}\end{array}\right.$

Thus, the equation to solve in order to obtain the initiation crack extension length $\lambda_{\mathrm{c}}$ is :

$\frac{1}{\tilde{\sigma}\left(\lambda_{c}\right)^{2}} \frac{B\left(\lambda_{c}\right)-B(0)}{\alpha \lambda_{c}^{2}}=\frac{1}{R} \frac{G_{\mathrm{c}}}{\sigma_{\mathrm{c}}^{2}}$

Leading to

$\left\{\begin{array}{l}K_{\mathrm{Ic}}^{\mathrm{app}}=\sqrt{\frac{\alpha \lambda_{c}^{2} G_{\mathrm{c}}}{B\left(\lambda_{c}\right)-B(0)}} \\ K_{\mathrm{IIIc}}^{\mathrm{app}}=\frac{\beta}{1-\beta} K_{\mathrm{Ic}}^{\mathrm{app}}\end{array}\right.$

Solving Eqs. 6 or 10 requires the computation of the normalized incremental energy release rate $\frac{B(\lambda)-B(0)}{\alpha \lambda^{2}}$, which is performed by FE and explained in next Section.

\section{FE modeling of daughter crack nucleation}

\subsection{FE model}

Solving the coupled criterion requires some FE element calculations in order to determine the stress fields prior to crack initiation and the potential energy change between undamaged and damaged states for various facet size. The mesh generation of the undamaged inner domain, whose geometry is depicted in Fig. 1a, is quite straightforward. A $2 \mathrm{D}$ mesh in the $y_{1} y_{2}$ plane is generated and extruded along the $y_{3}$ direction, the mesh 


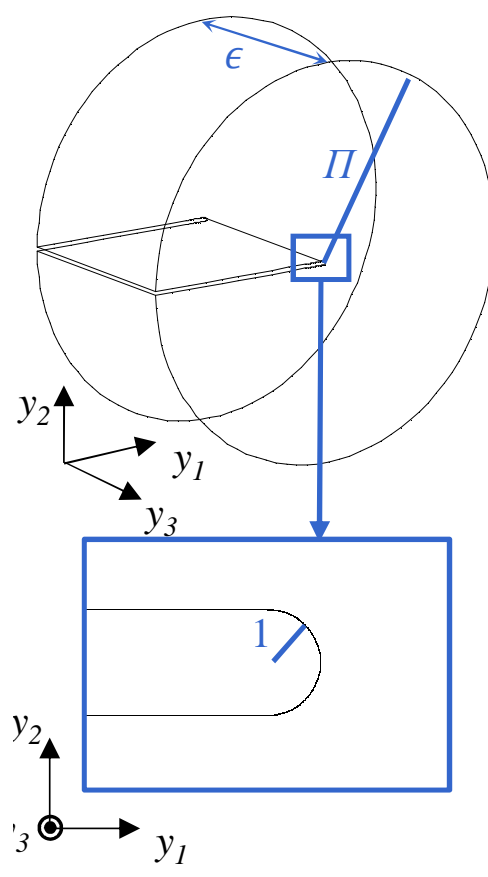

(a)

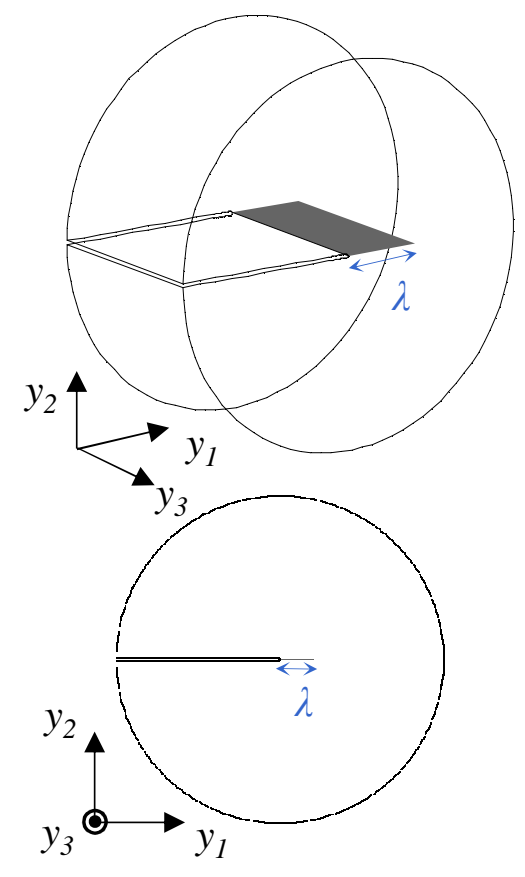

(b)

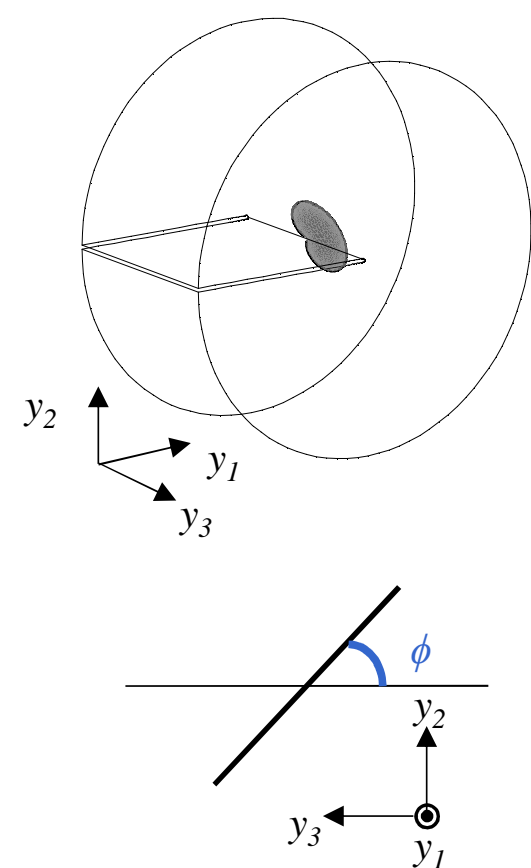

(c)

Fig. 1 (a) Inner domain of radius $\Pi$ and thickness $\epsilon$ considered around the parent crack blunted tip of radius 1 . Crack of length $l$ initiating ahead of the parent crack tip under (b) pure mode I and (c) mixed mode I+III loading.

being refined in the vicinity of the parent crack tip. Classical 15 nodes quadratic elements are used in order to compute the stress fields. While periodic boundary conditions are prescribed on the inner domain faces whose normal lies in the $y_{3}$-direction, displacements corresponding to a mode I+III far field loading around the sharp crack tip [31] of the outer domain are imposed on the fictitious boundary of the inner domain ( $c f$. Eq. 11). It is nothing more than the implementation of the matching conditions in the matched asymptotic expansions method (see the two first assumptions at the end of Section 2 and the Appendix).

$$
\left\{\begin{array}{l}
u_{1}=\frac{K_{\mathrm{I}}}{2 \mu} \sqrt{\frac{\rho}{2 \pi}} \cos \frac{\theta}{2}(3-4 \nu-\cos \theta) \\
u_{2}=\frac{K_{\mathrm{I}}}{2 \mu} \sqrt{\frac{\rho}{2 \pi}} \sin \frac{\theta}{2}(3-4 \nu-\cos \theta) \\
u_{3}=\frac{2 K_{\mathrm{III}}}{\mu} \sqrt{\frac{\rho}{2 \pi}} \sin \frac{\theta}{2}
\end{array}\right.
$$

where $(\rho, \theta)(\rho=r / R)$ are the polar coordinates in $y_{1} y_{2}$ plane of the inner domain, $K_{\mathrm{I}}$ and $K_{\mathrm{III}}$ the stress intensity factors independent of $y_{3}$ as a main assumption (Section 2), and $\mu$ the second Lamé coefficient (shear modulus). The FE calculations have been performed using Zset FE solver [34]. The material properties used for the calculations are $E=2.1 \mathrm{GPa}, \nu=0.395, G_{\mathrm{c}}=$ $8810^{-3} \mathrm{MPa} \mathrm{mm}$ and $\sigma_{\mathrm{c}}=35 \mathrm{MPa}$, which are repre- sentative of a polymer such as Homalite-100 studied by Pham and Ravi-Chandar [23,24].

The calculation of the potential energy change between undamaged and damaged states requires the generation of meshes containing cracks. Therefore, it is necessary to determine the possible initiation crack shape. If no assumptions are made on the $3 \mathrm{D}$ crack shape, it can possibly be described by an infinite number of parameters $[7,30]$, which complexifies the initiation crack shape determination. Doitrand and Leguillon $[9,10]$ recently overcome this difficulty by determining the possible crack shapes based on the stress isocontours, so that the $3 \mathrm{D}$ crack can be described by a single parameter. The same approach is used herein in order to determine the possible crack shapes in the plane maximizing the tensile stress at the blunted crack tip. Fig. 2a-b shows the normal stress field and isocontours in this plane for a ratio $\beta=0.37$. From a qualitative point of view, the stress isocontours exhibit a shape that is similar to that observed experimentally by Pham and Ravi-Chandar [24] (cf. Fig. 2c-d). Therefore, it seems reasonable to select the possible crack shapes based on the stress isocontours.

Once the possible crack shapes are determined, it is necessary to generate 3D meshes containing such cracks in order to compute the potential energy difference for 
(a)

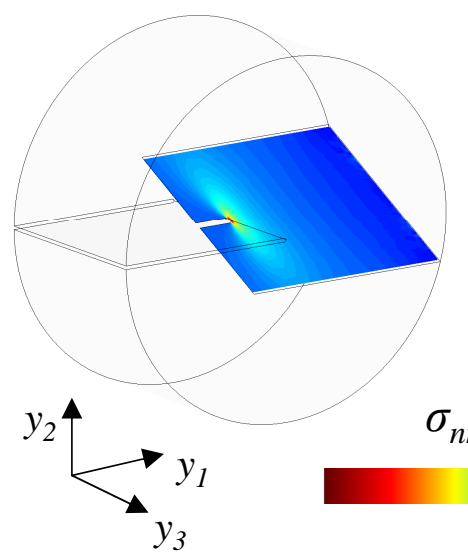

(c)

(b)

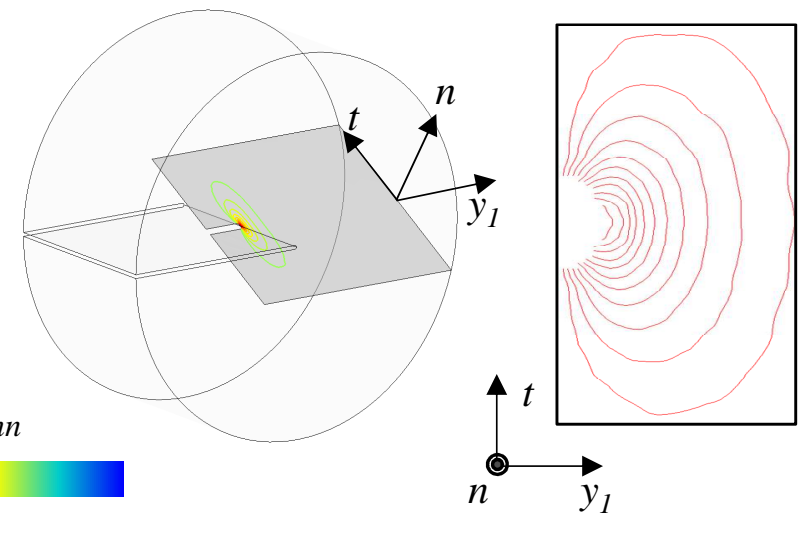

(d)

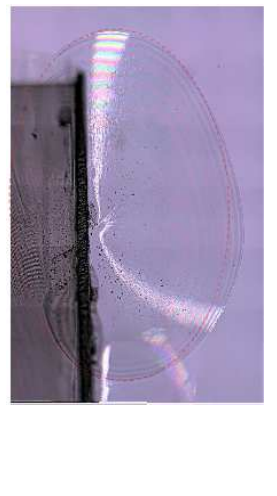

Fig. 2 Normal stress (a) field and (b) isocontours in the plane maximizing the tensile stress at the blunted crack tip. (c) Possible crack shapes based on the stress isocontours and (d) crack shape observed experimentally (Reprinted from [24]).

various crack surfaces. First, a 2D mesh of the cross section of the inner domain containing the projection of the crack outline in the plane $\left(O y_{1} y_{2}\right)$ is generated (Fig. 3a-b). Then, the mesh is extruded so as to obtain a $3 \mathrm{D}$ mesh which is geometrically sheared in the $\left(\mathrm{O}_{2} y_{3}\right)$ plane in order to get the crack back in the plane maximizing the tensile stress (Fig. 3c-e). The uncracked case is obtained from the cracked one by merging the double nodes lying on the opposite crack faces. Thus, the mesh topology is the same reducing the errors in the calculation of the difference in potential energy. It can be noted that the representative volume element faces in the $y_{3}$-direction are inclined with an angle corresponding to the crack angle. This is really convenient since it allows modeling an overlap between cracks, which would not be possible with vertical faces and a single inclined crack. Besides, the inner domain thickness $\epsilon$ in $y_{3}$-direction, which has no influence under pure mode I loading, now corresponds to the dimensionless spacing between the cracks in the modeled periodic network along $y_{3}$-direction. The determination of the initiation crack spacing is adressed in Section 5 .

\subsection{Inner domain size}

Although the inner domain is theoretically unbounded, to the aim of FE calculations it is artificially bounded by a fictitious boundary on which displacements are prescribed to represent the behavior at infinity. Therefore, the inner domain for the FE calculations must be large enough so that the blunted crack tip radius is small compared to the inner domain radius. In addition, it must also be large enough so that the area corresponding to the perturbation induced by the daugh- ter crack nucleation in the displacement field does not interact with the fictitious inner domain boundary.

In order to calibrate the inner domain size, the incremental energy release rate in pure mode I has been calculated for different inner domain radii $\Pi=50$, 100, 200, 400 (keep in mind the dimensionless crack tip radius is 1) and compared to the incremental energy release rate obtained using the method described by Leguillon et al. [19] ( $c f$. Fig. 4). The incremental energy release rate associated to a crack in an unbounded domain is a monotonic increasing function of the crack surface. For each inner domain radius, it is clear that if the crack extension length (in $y_{1}$ or $y_{2}$ direction, $c f$. Fig. 1) is larger than $\Pi / 20$, a non-monotonic incremental energy release rate is obtained, consequence of an interaction between the crack (that is too large compared to the inner domain size) and the outer fictitious boundary of the inner domain. However, if the crack extension is lower than $\Pi / 25$, the difference on the incremental release rate is lower than $1 \%$ compared to the reference obtained in [19]. Therefore, in the following, all the calculations have been performed ensuring an inner domain radius corresponding to at least 25 crack extensions along $y_{1}$ and $y_{2}$ direction.

\subsection{Pure mode I solution}

FE calculations using the inner domain presented in Fig 1a have been performed under pure mode I loading. The inner domain thickness (which has no influence on the results for pure mode I since the crack extends in the $0 y_{1} y_{3}$ plane), was choosen equal to 50 dimensionless crack tip radius. The incremental energy release rate has been calculated for several normalized crack length $\lambda$ (crack depicted in Fig. 1b). Then, by solving Eq. 5 for 


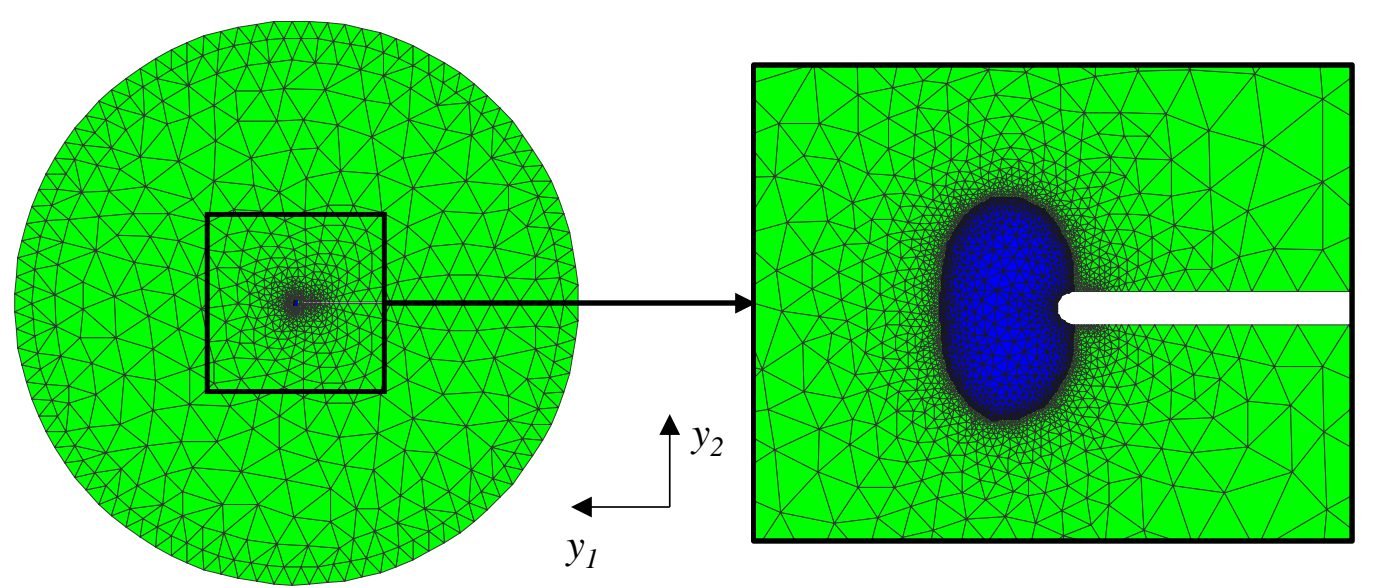

(a)

(b)
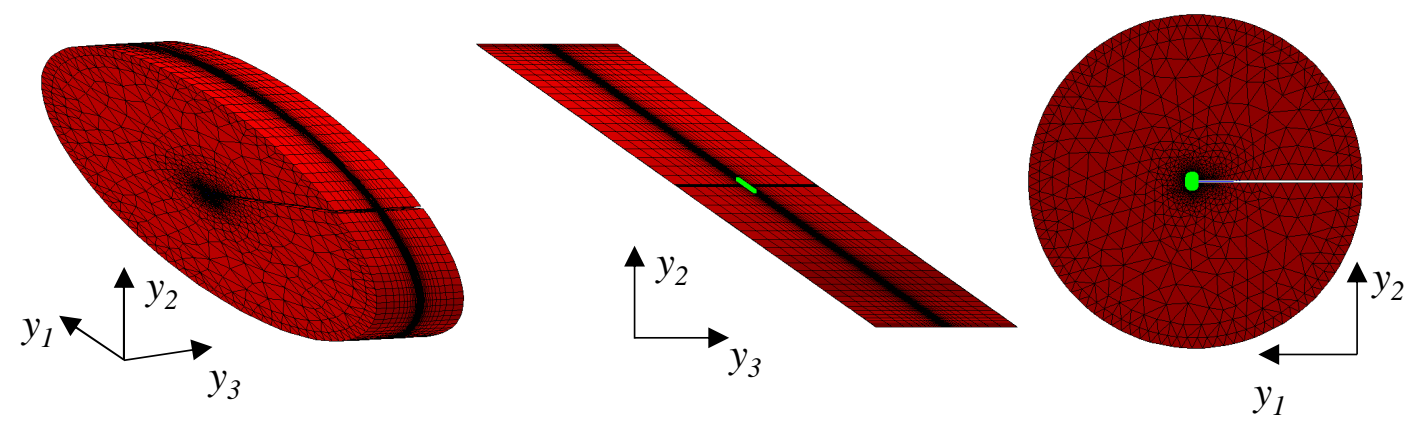

Fig. 3 (a) 2D mesh containing the 3D crack projection in the (Oxy) plane and (b) focus around the crack. (c) 3D and (d-e) lateral views of the $3 \mathrm{D}$ mesh obtained by extrusion and shearing of the $2 \mathrm{D}$ mesh.

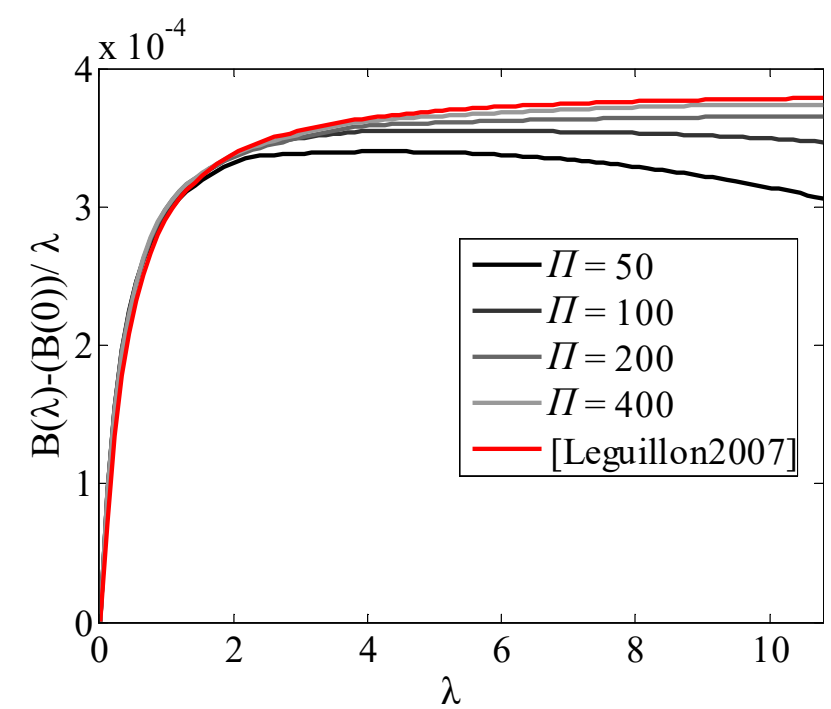

Fig. 4 Incremental energy release rate as a function of the dimensionless crack length under pure mode I loading obtained for different inner domain radius (the blunted crack tip radius is 1) and using [19].

different values of blunted crack tip radius $R$, the corresponding initiation normalized crack length has been determined, as well as the initiation load (given by Eq.
6 ). The initiation normalized length and loading as a function of the crack tip radius are presented in Fig. 5a and b. For instance, a crack tip radius $R=8910^{-3} \mathrm{~mm}$ ([24]) leads to an initiation crack length $56.210^{-3} \mathrm{~mm}$ at a loading level $K_{\mathrm{Ic}}^{\mathrm{app}}=0.58 \mathrm{MPa} \mathrm{m}^{1 / 2}$. As a matter of comparison, using [19], an initiation crack length of $55.310^{-3} \mathrm{~mm}$ and a loading level $K_{\mathrm{Ic}}^{\text {app }}=0.59 \mathrm{MPa}$ $\mathrm{m}^{1 / 2}$ are obtained for pure mode I loading, which shows the consistency of the proposed 3D FE modeling.

\subsection{Mixed mode I+III solution}

FE calculations using the inner domain presented in Fig 1c have been performed under mode I+III loading. The incremental energy release rate has been calculated for several crack spacing (e.g. the crack depicted in Fig. 1c and 3). The initiation normalized crack surface and loadings were then obtained by solving Eq. 9 and using Eqs. 10 and 7. Fig. 6 shows the initiation normalized crack surface and loading as a function of the blunted crack tip radius for an inner domain thickness $\epsilon$ corresponding to 10 times the dimensionless crack tip radius and a ratio $\beta=0.44$. A crack tip radius $R=8910^{-3}$ 


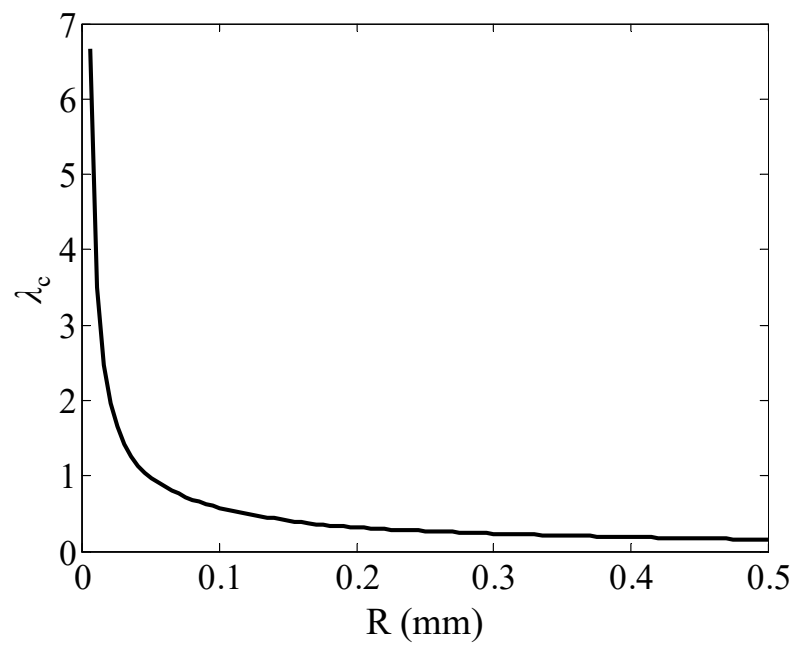

(a)

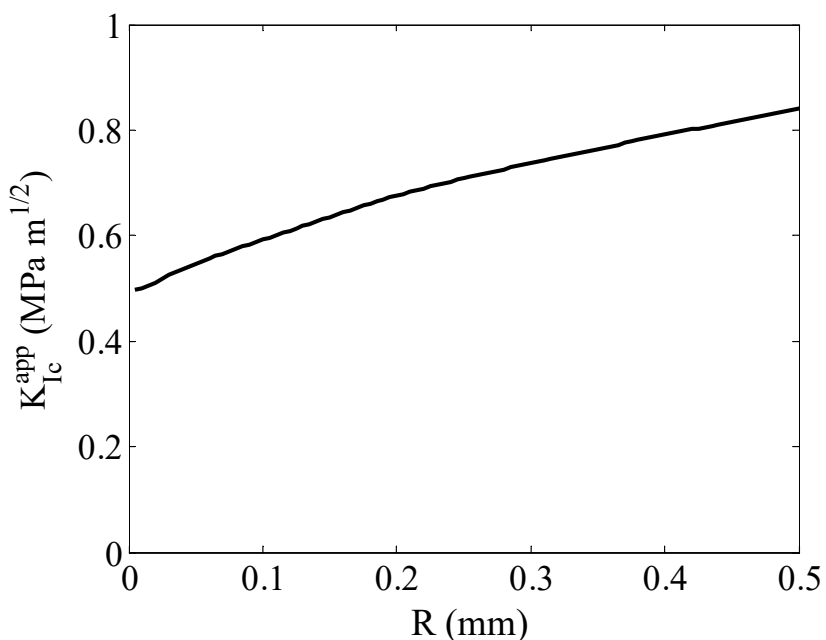

(b)

Fig. 5 Initiation (a) Normalized crack length and (b) loading as a function of blunted crack tip radius under pure mode I loading.

$\mathrm{mm}([24])$ leads to an initiation crack surface $3.04 \mathrm{~mm}^{2}$ at a loading level $K_{\text {Ic }}^{\text {app }}=0.71 \mathrm{MPa} \mathrm{m}^{1 / 2}$.

\section{Numerical results}

5.1 Comparison with experiments by Pham and Ravi-Chandar [23] and Chen et al. [3]

Fig. 7 shows the crack initiation angle as a function of the mode mixity ratio $\beta$ obtained experimentally by Pham and Ravi-Chandar [24] and numerically by FE. It seems that determining the crack orientation based on the maximum tensile stress ahead of the parent crack tip provides a good estimate of the crack initiation angle compared to experimental results. The proposed model allows determining the initiation crack surface and loading for a given crack spacing. Therefore, the initiation loading was determined as a function of the crack spacing to crack tip radius ratio $\epsilon=e / R$, which is presented in Fig. 8 for a mode mixity ratio $\beta=0.37$. It can be higlighted that the loading necessary to initiate a crack decreases with increasing crack spacing until a critical crack spacing above which it reaches a constant value. Indeed, there is a shielding effect that prevent the initiation of two cracks that are too close to each other. However, as soon as the crack spacing is sufficiently high, the cracks no longer interact so that the same loading level is obtained. Leguillon et al. [18] encountered the same situation in the case of multi-cracking in brittle thin layers and showed that the crack spacing at initiation may be determined as the mimimum spacing among those corresponding to the constant initiation loading. Repeating the same procedure for other mode mixity ratio $\beta$ between 0.17 and 1 , the initiation loadings $K_{\text {Ic }}^{\text {app }}$ and $K_{\text {IIIc }}^{\text {app }}$ can be determined ( $c f$. Fig. 9$)$. The large gap between $\beta=0$ and $\beta>0$ may be explained by two different mechanisms, continuous crack growth in the former case and fragmentation of the crack front in the latter.

In addition, the initiation crack spacing were found to lie between $0.44 \mathrm{~mm}$ and $0.51 \mathrm{~mm}$ for a blunted crack tip radius $R=8910^{-3} \mathrm{~mm}$, corresponding roughly to 5 crack tip radii. Therefore, it seems that the initiation crack spacing only depends on the parent crack tip dimensions, which is in agreement with Pham and RaviChandar conclusions [23]. Moreover, the crack spacing determined numerically is of the same order of magnitude as the one found experimentally by Pham and Ravi-Chandar [23] for Homalite-100, who measured an initiation crack spacing corresponding to around 4 parent crack tip radius. Similar order of magnitude was also obtained by Chen et al., who measured crack spacings in PMMA between 0.13 and $0.48 \mathrm{~mm}$, however, no indication was given about the crack tip radius.

5.2 Comparison with experiments by Pham and Ravi-Chandar [24]

Pham and Ravi-Chandar [24] designed a test so as to load a specimen in mode I+III through the combination of a wedge load and of a confining load resulting in a global compressive stress field ahead of the crack front. This particular set up promoted the sequential initiation of several cracks (at nearly similar loading levels) rather than a simultaneous initiation of these cracks. Moreover, an unstable propagation of the cracks just 


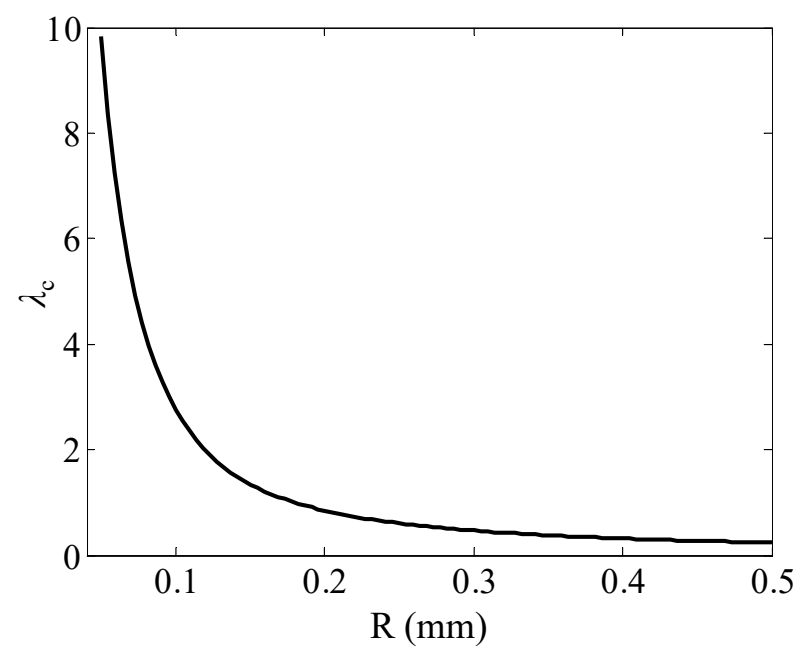

(a)

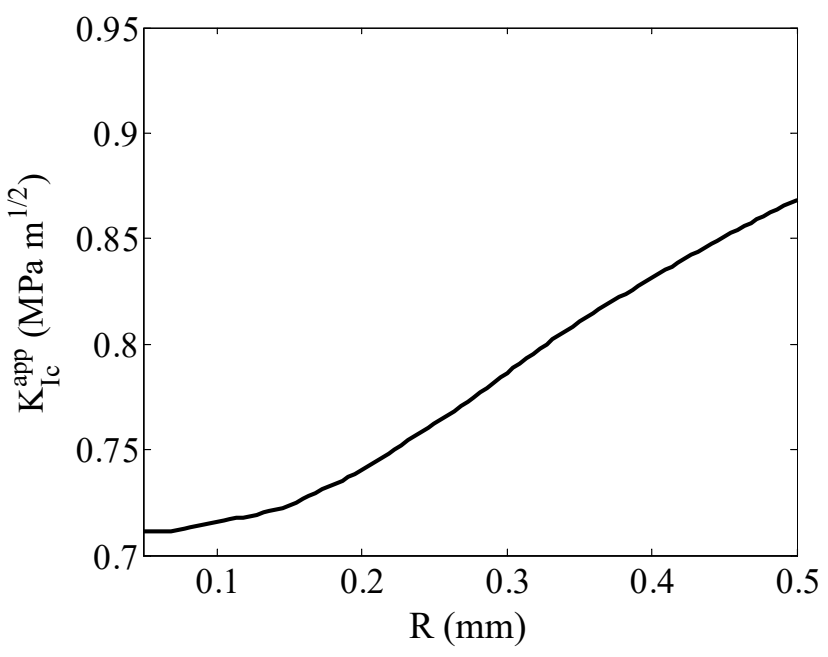

(b)

Fig. 6 Initiation (a) Normalized crack surface and (b) loading as a function of blunted crack tip radius under mode I+III loading with a mode mixity ratio $\beta=0.44$.

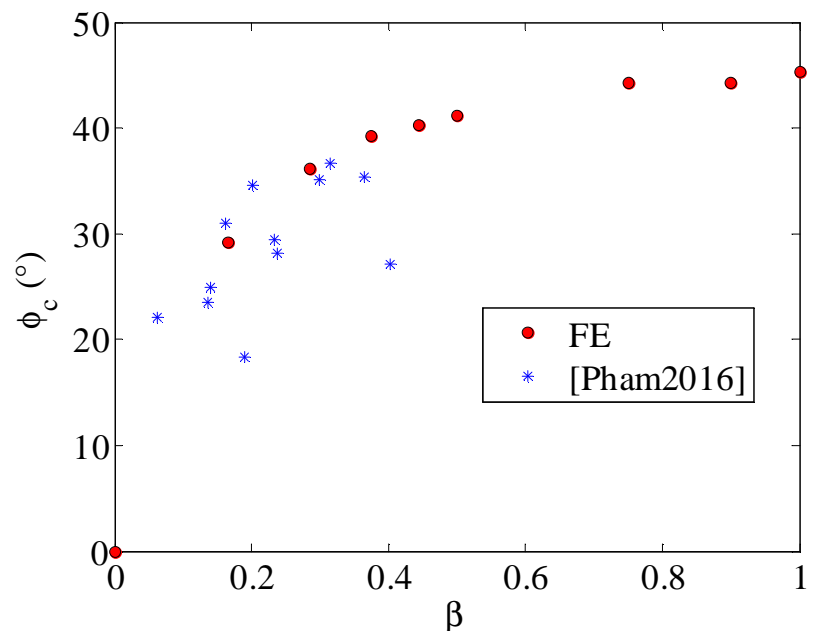

Fig. 7 Crack initiation angle as a function of the mode mixity ratio $\beta$ obtained experimentally by Pham and RaviChandar [24] and numerically by FE.

after initiation was observed. Therefore, the first crack likely reached a bigger size than in the case of simultaneous crack initiations [23] . This phenomenon highlights the difficulty to precisely measure an initiation length in certain experiments. Indeed, in the present case, the initiation length $l_{\mathrm{c}}$ is not an arrest length since an unstable growth is observed rigth after crack initiation. This can be explained considering the relation between the differential $(G)$ and incremental $\left(G^{i n c}\right)$ release rates (cf. Eq. 12) [8].

$G(S)=\frac{d G^{i n c}(S)}{d S} S+G^{i n c}(S)$

At crack initiation, $G^{i n c}=G^{c}$, therefore it is clear that for a strictly monotonic increasing evolution of $G^{i n c}$,

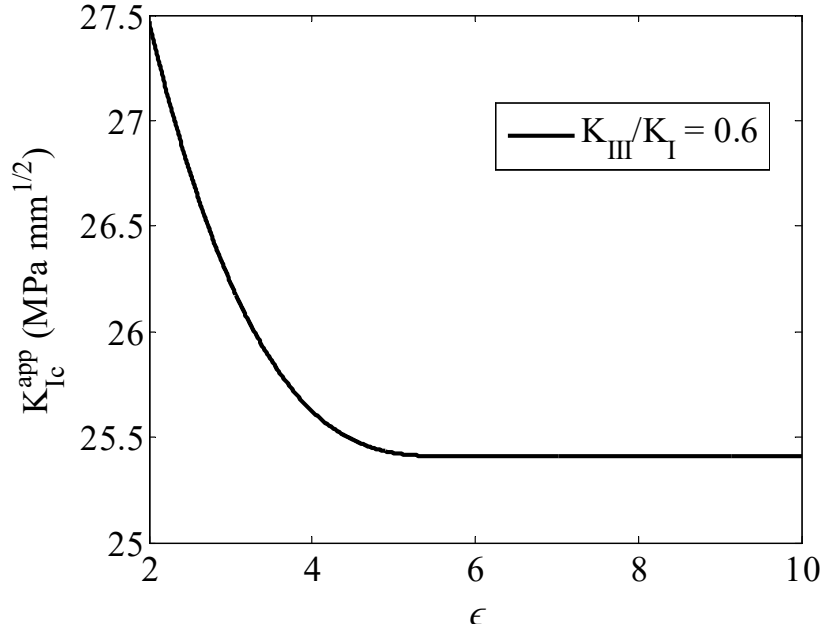

Fig. 8 Crack initiation loading as a function of the crack spacing obtained for a mode mixity ratio $\beta=0.37$.

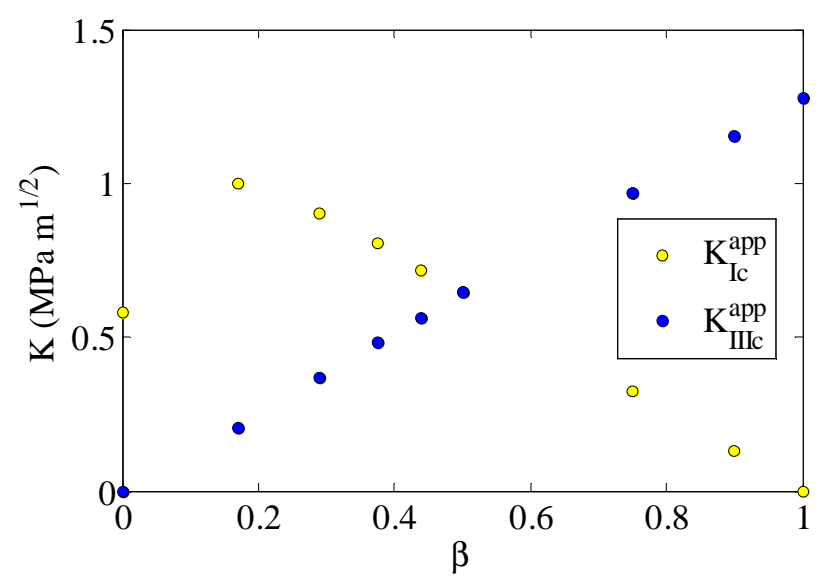

Fig. 9 Initiation loadings $K_{\text {Ic }}^{\text {app }}$ and $K_{\text {IIIc }}^{\text {app }}$ as a function of the mode mixity ratio $\beta$. 


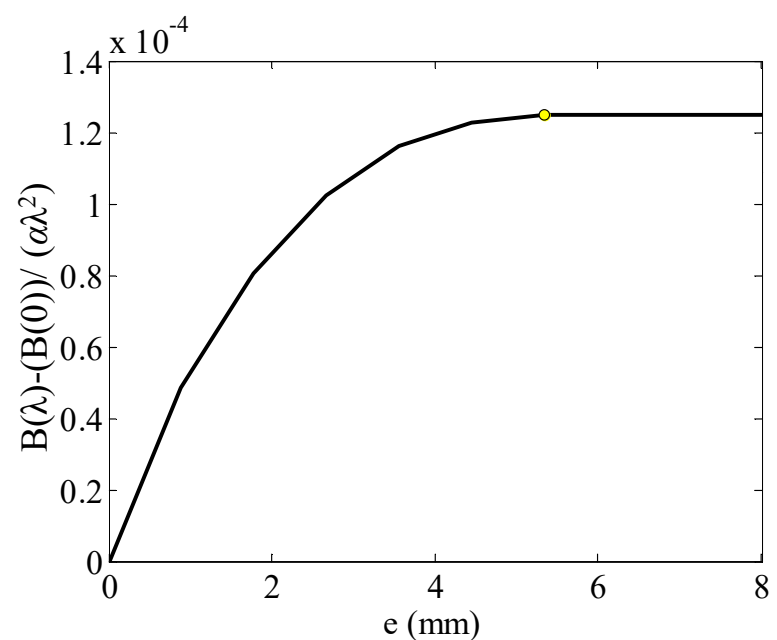

Fig. 10 Normalized incremental energy release rate as a function of the crack spacing obtained for a mode mixity ratio $\beta=0.37$ under the assumption that the first crack has propagated in an unstable maneer just after initiation.

$G(S)>G^{i n c}(S)=G^{c}$, resulting in unstable crack propagation immediatly following the initiation. A spacing between cracks ranging from 4.9 to $6.6 \mathrm{~mm}$ was measured experimentally, both cracks exhibiting nearly the same size (crack major and minor axes were about 6.3 and $3.7 \mathrm{~mm}$ assuming a nearly elliptic shape) and angles (between 29 and $36 \mathrm{deg}$.). We apply herein the model presented in Section 4.1 assuming that the crack shape is fixed with dimensions close to those measured in [24], with a tilt angle $\phi=33 \mathrm{deg}$. for various crack spacings. Fig. 10 shows the normalized incremental energy release rate as a function of the crack spacing. Similarly to the results obtained previously, the initiation crack spacing can be determined as the minimum spacing among those corresponding to a constant normalized incremental energy release rate (or a constant initiation loading). A initiation crack spacing of $5.3 \mathrm{~mm}$ is obtained, which is in good agreement with the spacings measured experimentally [24].

\section{Conclusion}

Adressing crack propagation under mode I+III loading requires studying the parent crack front segmentation in multiple facets that grow and later on coalesce. The crack front segmentation thus looks like a crack initiation problem rather than a problem of crack growth. The proposed approach, which is a new application of the coupled criterion to the tridimensional case, allows the nucleation of facets ahead of a parent crack under mode I+III loading to be simulated. Crack initiation shape, orientation and spacing is determined for any mode mixity ratio by coupling a stress and an en- ergy criterion in the framework of matched asymptotic expansions. Crack orientation is determined based on the maximum tensile stress ahead of the parent crack tip. The obtained crack angles for several mode mixity ratios show a good agreement with experimental results. Crack initiation size, loading and spacing are dependent on the blunted crack tip radius. Similar crack spacings are obtained whatever the mode mixity ratio, which are of the same order or magnitude as crack spacings measured experimentally. The possible crack shapes based on the stress isocontours are qualitatively close to those observed experimentally, which indicates the consistency of determining the possible cracks using these isocontours. It is worth highlighting the convenience of such a method, which allows parameterizing the cracks by a single variable. The only assumptions made on the crack shape is that it initiates in a plane, which is representative of the cracks observed experimentally except for their tips, which are gently curved. A possible improvement of the proposed method would be the determination of the 3D crack shape without making the assumption of a plane extension.

\section{Appendix}

\section{The matched asymptotic expansions}

The actual domain embeds the parent blunted crack (root radius $R$ ) and a group of small slanted crack regularly spaced. The outer domain is obtained for $R \rightarrow 0$, and as a consequence $l \rightarrow 0$ since it is assumed that $l$ is smaller or of the same order of magnitude than $R$ (to be checked afterwards). In the outer domain, the blunted crack becomes a slit with no thickness. Both actual and outer domains are spanned by the Cartesian space variables $x_{i}(i=1,3)\left(r, \theta, x_{3}\right.$, in cylindrical coordinates).The outer expansion with respect to the small parameter $R$ can be written $\left(\nabla_{x}\right.$ is the gradient operator with respect to the $x_{i}$ 's)

$$
\left\{\begin{array}{l}
\underline{U^{R}}\left(x_{1}, x_{2}, x_{3}, l\right)=\underline{U}^{0}\left(x_{1}, x_{2}, x_{3}\right)+\ldots \\
\underline{\underline{\sigma}}\left(\underline{U}^{R}\right)=\mathbf{C}: \nabla_{x} \underline{U^{R}}=\mathbf{C}: \nabla_{x} \underline{U}^{0}+\ldots
\end{array}\right.
$$

The dots denote a remainder that is small and decreases with $R$. The behavior of the leading term of Eq. (13) near the crack tip is described by the Williams series (polar and Cartesian coordinates are mixed without risk of confusion)

$$
\begin{aligned}
\underline{U}^{0}\left(x_{1}, x_{2}, x_{3}\right)= & \underline{C}+K_{\mathrm{I}} \sqrt{r} \underline{u}_{\mathrm{I}}(\theta)+K_{\mathrm{III}} \sqrt{r} \underline{u}_{\mathrm{III}}(\theta)+\ldots \\
= & \underline{C}+K_{\mathrm{I}} \sqrt{r}\left[\underline{u}_{\mathrm{I}}(\theta)+m \underline{u}_{\mathrm{III}}(\theta)\right]+\ldots \\
& \text { with } m=\frac{K_{\mathrm{III}}}{K_{\mathrm{I}}}=\left(\frac{1}{\beta}-1\right)^{-1}
\end{aligned}
$$


The leading term is an irrelevant constant (rigid translation), $K_{\mathrm{I}}$ and $K_{\mathrm{III}}$ are the modes I and III stress intensity factors and $\underline{u}_{\mathrm{I}}$ and $\underline{u}_{\mathrm{III}}$ are the associated opening and shear modes. The intensity factors $K_{\mathrm{I}}$ and $K_{\mathrm{III}}$ are independant of $x_{3}$ as a consequence of the first assumption stated at the end of Section 2 .

The inner domain is obtained by zooming in the actual domain by $1 / R$ and considering again the limit as $R \rightarrow 0$. It is an unbounded domain spanned by the dimensionless space variables $y_{i}=x_{i} / R(i=1,3)$; it embeds the semi-infinite parent crack and an infinite number of regularly spaced slanted cracks with length $\lambda=l / R$ along $y_{1}$ axis. In the $y_{3}$ direction, a periodicity assumption is done reducing the domain to a single period of length $\epsilon=e / R$ in this direction (see Figures 1 to 3$)$.

After having fulfilled the matching conditions [20], the inner expansion can be written

$$
\begin{aligned}
\underline{U}^{R}\left(x_{1}, x_{2}, x_{3}, l\right) & =\underline{U}^{R}\left(R y_{1}, R y_{2}, R y_{3}, R \lambda\right) \\
& =\underline{C}+K_{\mathrm{I}} \sqrt{R} \underline{\underline{I}}_{\mathrm{I}}\left(y_{1}, y_{2}, y_{3}, \lambda\right) \\
& +K_{\mathrm{III}} \sqrt{R} \underline{V}_{\mathrm{III}}\left(y_{1}, y_{2}, y_{3}, \lambda\right)+\ldots \\
& =\underline{C}+K_{\mathrm{I}} \sqrt{R}\left[\underline{V}_{\mathrm{I}}\left(y_{1}, y_{2}, y_{3}, \lambda\right)\right. \\
& \left.+m \underline{V}_{\mathrm{III}}\left(y_{1}, y_{2}, y_{3}, \lambda\right)\right]+\ldots
\end{aligned}
$$

where $\underline{V}_{\mathrm{I}}$ (respectively $\left.\underline{V}_{\mathrm{III}}\right)$ behaves like $\sqrt{\rho} \underline{u}_{\mathrm{I}}(\theta)$ (respectively $\left.\sqrt{\rho} \underline{u}_{\mathrm{III}}(\theta)\right)$ at infinity $(\rho=r / R \rightarrow \infty)$. Relationship (15) can be rewritten for the purpose of full FE calculations

$$
\left\{\begin{array}{l}
\underline{U}^{R}\left(x_{1}, x_{2}, x_{3}, l\right)=\underline{C}+K_{\mathrm{I}} \sqrt{R} \underline{W}\left(y_{1}, y_{2}, y_{3}, \lambda\right)+\ldots \\
\underline{\underline{\sigma}}\left(\underline{U}^{R}\right)=\frac{1}{R} \mathbf{C}: \nabla_{y} \underline{U}^{R}=\frac{K_{\mathrm{I}}}{\sqrt{R}} \mathbf{C}: \nabla_{y} \underline{W}=\frac{K_{\mathrm{I}}}{\sqrt{R}} \tilde{\underline{\sigma}}(\underline{W})
\end{array}\right.
$$

Where $\underline{W}=\underline{V}_{\mathrm{I}}+m \underline{V}_{\mathrm{III}}$ behaves at infinity like $\sqrt{\rho} \underline{u}_{\mathrm{I}}(\theta)+$ $m \sqrt{\rho} \underline{u}_{\mathrm{III}}(\theta)$. According to [19], the change in potential energy takes the form

$$
\begin{aligned}
-\delta W_{\mathrm{p}} & =\Psi_{x}\left(\underline{U}^{R}\left(x_{1}, x_{2}, x_{3}, l\right), \underline{U}^{R}\left(x_{1}, x_{2}, x_{3}, 0\right)\right) \\
& =\Psi_{x}\left(\underline{U}^{R}\left(x_{1}, x_{2}, x_{3}, l\right), \underline{U}^{0}\left(x_{1}, x_{2}, x_{3}\right)\right) \\
& -\Psi_{x}\left(\underline{U}^{R}\left(x_{1}, x_{2}, x_{3}, 0\right), \underline{U}^{0}\left(x_{1}, x_{2}, x_{3}\right)\right)
\end{aligned}
$$

where

$\Psi_{x}(\underline{F}, \underline{G})=\frac{1}{2} \int_{\Gamma_{x}}[\underline{\underline{\sigma}}(\underline{F}) \cdot \underline{n} \cdot \underline{G}-\underline{\underline{\sigma}}(\underline{G}) \cdot \underline{n} \cdot \underline{F}] \mathrm{d} s$

The contour $\Gamma_{x}$ starts from the stress free edges of the crack and embeds the blunted notch and the crack extension. The integral (18) is path indepedent for any two functions $\underline{F}$ and $\underline{G}$ fulfilling the equilibrium equations. The index $x$ in Eqs. (17) and (18) recalls that the calculations are a priori carried out in the actual (using the actual solution) or the outer domain (using the outer expansion but this requires calculating the remainder in 13 [16]).
Taking into account the change of variables defined by the dilatation in the inner domain, (18) rewrites

$$
\begin{aligned}
\Psi_{x}(\underline{F}, \underline{G}) & =\frac{R}{2} \int_{\Gamma_{y}}[\underline{\underline{\tilde{\sigma}}}(\underline{F}) \cdot \underline{n} \cdot \underline{G}-\underline{\underline{\sigma}}(\underline{G}) \cdot \underline{n} \cdot \underline{F}] \mathrm{d} S \\
& =R \Psi_{y}(\underline{F}, \underline{G})
\end{aligned}
$$

Because $\mathrm{d} S=R^{2} \times \mathrm{d} s$ (while $\mathrm{d} S=R \times \mathrm{d} s$ in 2 D leading to two similar relationships expressed either with respect to the $x_{i}$ 's or the $y_{i}$ 's). Then

$$
\begin{aligned}
-\delta W_{\mathrm{p}} & =K_{\mathrm{I}}^{2} R^{2}\left[\Psi_{y}\left(\underline{W}\left(y_{1}, y_{2}, y_{3}, \lambda\right), \sqrt{\rho}\left[\underline{u}_{\mathrm{I}}(\theta)+m \underline{u}_{\mathrm{III}}(\theta)\right]\right)\right. \\
& -\Psi_{y}\left(\underline{W}\left(y_{1}, y_{2}, y_{3}, 0\right), \sqrt{\rho}\left[\underline{u}_{\mathrm{I}}(\theta)+m \underline{u}_{\mathrm{III}}(\theta)\right]\right) \\
& =K_{\mathrm{I}}^{2} R^{2}(B(\lambda)-B(0))+\ldots
\end{aligned}
$$

Note that $B(\lambda)-B(0)$ is nothing but the change in potential energy computed in the $y_{i}$ variables when moving from $\underline{W}\left(y_{1}, y_{2}, y_{3}, 0\right)$ to $\underline{W}\left(y_{1}, y_{2}, y_{3}, \lambda\right)$.

With a newly created crack surface $S=\alpha l^{2}(\alpha$ is a scaling coefficient) it comes the energy condition

$G_{\mathrm{inc}}=-\frac{\delta W_{\mathrm{P}}}{S}=K_{\mathrm{I}}^{2} \frac{B(\lambda)-B(0)}{\alpha \lambda^{2}} \geq G_{\mathrm{c}}$

Denoting $\sigma$ and $\tilde{\sigma}$ the tensile component of the corresponding stress tensors, the stress condition can be written

$\sigma\left(\underline{U}^{R}\right)=\frac{K_{\mathrm{I}}}{\sqrt{R}} \tilde{\sigma}(\underline{W}) \geq \sigma_{\mathrm{c}}$

here $\tilde{\sigma}(\underline{W})$ is nothing but the tensile stress computed in the $y_{i}$ variables.

Combining (21) and (22) gives the equation for the dimensionless crack extension length $\lambda_{\mathrm{c}}$

$\frac{1}{\tilde{\sigma}\left(\lambda_{\mathrm{c}}\right)} \frac{B(\lambda)-B(0)}{\alpha \lambda_{\mathrm{c}}^{2}}=\frac{1}{R} \frac{G_{\mathrm{c}}}{\sigma_{\mathrm{c}}^{2}}$

In this equation $\tilde{\sigma}\left(\lambda_{\mathrm{c}}\right)$ denotes the tensile stress associated with $\underline{W}$, expressed in the $y_{i}$ variables and computed at a dimensionless distance $\lambda_{\mathrm{c}}$ of the notch root along the $y_{1}$ axis. Then the load at failure can be derived from $(21)$

$K_{\mathrm{Ic}}^{\mathrm{app}}=\sqrt{\frac{\alpha \lambda_{\mathrm{c}}^{2} G_{\mathrm{c}}}{B\left(\lambda_{\mathrm{c}}\right)-B(0)}} ; K_{\mathrm{IIIc}}^{\mathrm{app}}=m K_{\mathrm{Ic}}^{\mathrm{app}}$ 


\section{References}

1. Buchholz FB, Chergui A, Richard HA (2004) Fracture analyses and experimental results of crack growth under general mixed mode loading conditions. Eng Fract Mech $71: 455-468$

2. Cambonie T, Lazarus V (2014) Quantification of the crack fragmentation resulting from mode I+III loading. Procedia Materials Science 3:1816-1821

3. Chen $\mathrm{CH}$, Cambonie T, Lazarus V, Nicoli M, Pons AJ, Karma A (2015) Crack Front Segmentation and Facet Coarsening in Mixed-Mode Fracture. Phys Rev Lett 115:265503

4. Citarella R, Buchholz FG (2008) Comparison of crack growth simulation by DBEM and FEM for SEN specimens undergoing torsion or bending loading. Eng Fract Mech 75:489-509

5. Cox SJD, Scholz CH (1988) On the formation and growth of faults: an experimental study. J Struct Geol 10:413-430

6. Dhondt G, Chergui A, Buchholz FG (2001) Computational fracture analysis of different specimens regarding 3D and mode coupling effects. Eng Fract Mech 68:383-401

7. Doitrand A, Fagiano C, Carrère N, Chiaruttini V, Hirsekorn M (2017) Damage onset modeling in woven composites based on a coupled stress and energy criterion. Eng Fract Mech 169:189-200

8. Doitrand A, Fagiano C, Hild F, Chiaruttini V, Mavel A, Hirsekorn M (2017) Mesoscale analysis of damage growth in woven composites energy criterion. Compos Part A 96:7788

9. Doitrand A, Leguillon D (2018) 3D application of the coupled criterion to crack initiation prediction in epoxy/aluminum specimens under four point bending. Int J Sol Struct: in press

10. Doitrand A, Leguillon D (2018) Comparison between 2D and $3 \mathrm{D}$ applications of the coupled criterion to crack initiation prediction in scarf adhesive joints. Int J Adhesion Adhesives: under review

11. Eberlein A, Richard HA, Kullmer G (2017) Facet formation at the crack front under combined crack opening and anti-plane shear loading. Eng Fract Mech 174:21-29

12. Lazarus V, Buchholz FG, Fulland M, Wiebesiek J (2008) Comparison of predictions by mode II or mode III criteria on crack front twisting in three or four point bending experiments. Int J Fract 153:141-151

13. Leblond JB, Karma A, Lazarus V (2011) Theoretical analysis of crack front instability in mode I+III. J mech Phys Solids 59:1872-1887

14. Leblond JB, Lazarus V, Karma A (2015) Multiscale cohesive zone model for propagation of segmented crack fronts in mode I+III fracture. Int J Fract 191:167-189

15. Leguillon, D (2002) Strength or toughness? A criterion for crack onset at a notch. Eur J Mech - A/Solids 21:61-72

16. Leguillon D (2011) Determination of the length of a short crack at a v-notch from a full field measurement. Int. J. Solids Structures 48:884-892.

17. Leguillon D (2014) An attempt to extend the 2D coupled criterion for crack nucleation in brittle materials to the $3 \mathrm{D}$ case. Theor Appl fract mech 74:7-17

18. Leguillon D, Li J, Martin E (2017) Multi-cracking in brittle thin layers and coatings using a FFM model. Eur J Mech A/Sol 63:14-21

19. Leguillon D, Quesada D, Putot C, Martin E (2007) Prediction of crack initiation at blunt notches and cavities size effects. Eng Fract Mech 74:2420-2436
20. Leguillon D, Sanchez-Palencia E (1987) Computation of singular solutions in elliptic problems and elasticity, John Wiley \& Son, New York, and Masson, Paris

21. Leguillon D, Yosibash Z (2003) Crack onset at a v-notch. Influence of the notch tip radius. Int J Fract 122:1-21

22. Mittelman B, Yosibash Z (2015) Energy release rate cannot predict crack initiation orientation in domains with a sharp V-notch under mode III loading. Eng Fract Mech 141:230-241

23. Pham KH, Ravi-Chandar K (2014) Further examination of the criterion for crack initiation under mixed mode I+III loading. Int J Fract 189:121-138

24. Pham KH, Ravi-Chandar K (2016) On the growth of cracks under mixed-mode I+III loading. Int J Fract 199:105-134

25. Pollard DD, Segall P, Delaney PT (1982) Formation and interpretation of dilatant echelon cracks. Geol Soc Am Bull 93:1291-1303

26. Pons AJ, Karma A (2010) Helical crack-front instability in mixed-mode fracture. Nature 464:85-89

27. Picard D, Leguillon D, Putot C (2006) A method to estimate the influence of the notch-root radius on the fracture toughness measurement of ceramics. J Eur Cer Soc 26:14211427

28. Knauss WG (1970) An observation of crack propagation in antiplane shear. Int J Fract 6:183-187

29. Sommer E (1969) Formation of fracture lances in glass. Eng Fract Mech 1:539-546

30. Weißgraeber P, Leguillon D, Becker W (2016) A review of Finite Fracture Mechanics: crack initiation at singular and non-singular stress raisers. Archive Appl Mech 86:375-401

31. Westergaard HM (1934) Stresses at a crack, size of the crack and the bending of reinforced concrete. Proc American Concrete Institute 30:93-102

32. Yosibash Z, Mittelman B (2016) A 3-D failure initiation criterion from a sharp V-notch edge in elastic brittle structures. Eur J Mech A/Sol 60:70-94

33. Younes AI, Engelder T (1999) Fringe cracks: key structures for the interpretation of the progressive Alleghanian deformation of the Appalachian plateau. GSA Bull 111:219239

34. http://www.zset-software.com/products/zebulon/ 